\title{
Head Trauma in Infants: Experience of a High Flow Tertiary Hospital in a Developing Country
}

\author{
Mohamed M. Salama, Ahmed El-Fiki \\ Department of Neurosurgery, Faculty of Medicine, Cairo University, Giza, Egypt \\ Email: mosalama75@gmail.com, asfiki@yahoo.com
}

Received 17 February 2016; accepted 8 April 2016; published 11 April 2016

Copyright (C) 2016 by authors and Scientific Research Publishing Inc.

This work is licensed under the Creative Commons Attribution International License (CC BY). http://creativecommons.org/licenses/by/4.0/

(c) (i) Open Access

\begin{abstract}
Background and Objective: Head trauma in infants is a common health problem that has not been the focus of many research articles. The aim of study was to describe the epidemiology, etiology, management and clinical outcome of head trauma in infants ( $<2$ years) in a high flow tertiary hospital. Patients and Methods: This study was conducted on 95 consecutive infants with head trauma admitted to the neurotrauma unit in Cairo University hospitals during the period between September 2013 and December 2014. The data of these patients including age, sex, mode of trauma, neurological status on admission, CT findings, operative details in surgical cases, clinical outcome, and length of hospital stay were analyzed. Results: The study included 57 boys and 38 girls, with mean age of 13.2 months (range: 3 days - 23 months). The most common mode of trauma was falls (82.1\%). Most of the patients had mild head injury (84.2\%). Twenty two patients were operated upon, including 9 patients for evacuation of hematomas, 11 patients for elevation of depressed fractures, 1 patient for decompressive craniotomy and 1 patient for growing skull fracture. Good recovery followed in 83 patients. There were 4 deaths. Mean length of stay was 4 days (range: 1 31 days). Conclusion: Most of head injuries in infants are mild. The commonest etiology is falls, which is a preventable cause in many circumstances. The majority of these patients do not require surgical intervention. Good recovery is the rule in most of these infants with low mortality rates.
\end{abstract}

\section{Keywords}

Head Trauma, Infants, Traumatic Brain Injury

\section{Introduction}

Traumatic brain injury is a major cause of morbidity and mortality in infants and children [1] [2]. Although the 
primary traumatic brain injury is not amenable to treatment, except through prevention, management is directed at preventing or reducing the effects of secondary physiologic events and the secondary injuries that follow [2] [3]. Most of the cases are due to accidental injuries with only a little group suffering from nonaccidental injuries [4].

Traumatic brain injury in infants differs from adults in many aspects including the response to injury, brain plasticity and clinical outcome [5]. The mechanism of injury, its degree of severity, the occurrence of multiple trauma, and the extent of the secondary insults can all affect the outcome [3]. There are some surgical considerations regarding operating on infants with closed skull due to minimal space that can accommodate only mild increase in intracranial pressure. On the other hand, younger infants with open fontanels and patent sutures could better accommodate the rise in the intracranial pressure. Thin and delicate skin can be a problem in wound closure especially if closure is under tension [6].

Despite its importance, only few articles have addressed accidental head injury in infants as a separate entity. They found that most of these injuries were mild resulting from falls and carried a good prognosis in the majority of patients [7] [8]. The aim of this study was to describe the epidemiology, etiology, management and clinical outcome of head trauma in infants ( $<2$ years) in a high flow tertiary hospital in Egypt.

\section{Patients and Methods}

This prospective study was conducted on infants ( $<2$ years) with head trauma admitted to the neurotrauma unit in Cairo University hospitals during the period between September 2013 and December 2014. Infants with birth-related head trauma were excluded from the study. Infants who presented to the emergency room but were not admitted to the hospital were not included. There were no exclusion criteria regarding the clinical condition or associated injuries.

All infants were subjected to the first aid measures and detailed trauma survey was performed unless head trauma was isolated. Assessment of the neurological condition focused on conscious level assessment based on pediatric Glasgow coma scale (PGCS) [9] [10], and examination for signs of lateralization especially motor deficits and pupil irregularities. Examination for subgaleal hematomas or scalp wounds with exploration for any underlying fractures, and examination for bleeding or cerebrospinal fluid (CSF) leak from orifices were performed. Determining the severity of traumatic brain injury was based on the PGCS.

Computed tomography (CT) of the brain with bone window was the radiological modality performed for all admitted patients even those with mild trauma and no conscious level affection. Skull X-ray was performed for few patients with mild trauma and no clinical indication for CT and these patients were admitted to the hospital only if X-ray showed a fissure, where CT brain was subsequently performed before admission. All patients were admitted to the neurotrauma unit whether into the ward or the intensive care unit based on the clinical condition. Management plans were mainly based upon the traumatic brain injury management guidelines published in 2006 [11]. CT brain was repeated according to the initial CT findings and clinical condition. All patients who were operated upon had a follow up CT within 24 hours from surgery. Outcome was assessed at discharge according to the pediatric Glasgow outcome score (GOS-E Peds) [12].

The data of these patients including age, sex, mode of trauma, neurological status on admission and associated injuries, CT findings, operative details in surgical cases, clinical outcome, and length of hospital stay were documented and analyzed.

\section{Results}

The study included 95 infants with mean age of 13.2 months (range: 3 days - 23 months). Table 1 shows the demographics and the severity of head injury in our patients. The most common mode of trauma was falls in 78 patients (82.1\%), including fall from height in 61 patients, fall over stairs in 14 patients and fall to the ground in 3 patients. Other modes of trauma included trauma with blunt or sharp objects in 13 patients (13.7\%), road traffic accidents in 2 patients (2.1\%), and pellet injury in 2 patients (2.1\%).

Clinical examination revealed that 13 patients (13.7\%) had scalp wounds including 9 patients with underlying depressed fractures. Thirty one patients (32.6\%) had subgaleal hematomas which resolved within 2 weeks in all patients. Seven of these patients required blood transfusion due to drop of hemoglobin levels below $8 \mathrm{~g} / \mathrm{dL}$. Seven patients had CSF leak per orifices; 5 with leak from the nose and 2 with leak from the ear. Six patients (6.3\%) had associated injuries including lung contusions in 2 patients, intra-abdominal fluid collection in 2 
Table 1. The demographics and severity of head injury.

\begin{tabular}{ccc}
\hline & & Number (percentage) \\
\hline \multirow{2}{*}{ Sex } & Male & $57(60 \%)$ \\
& Female & $38(40 \%)$ \\
\multirow{3}{*}{ Age } & $<1$ month & $5(5.3 \%)$ \\
& $1-<6$ months & $15(15.8 \%)$ \\
& $6-<12$ months & $17(17.9 \%)$ \\
Severity & $12-<18$ months & $24(25.2 \%)$ \\
& $18-<24$ months & $34(35.8 \%)$ \\
& Mild (PGCS 13 - 15) & $80(84.2 \%)$ \\
& Moderate (PGCS 9 - 12) & $10(10.5 \%)$ \\
\hline
\end{tabular}

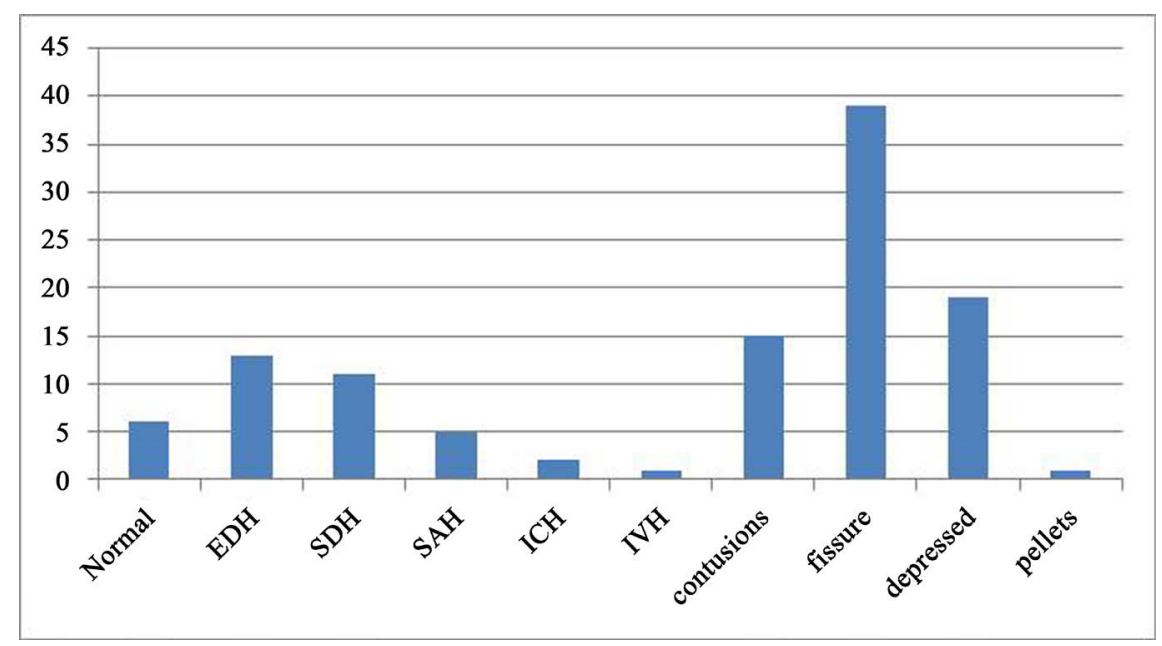

Figure 1. This figure shows the initial CT findings.

patients including a patient with liver tears, vitreous hemorrhage in one patient, and fracture femur in one patient.

Initial CT brain revealed a variety of lesions including extradural hematoma (EDH), subdural hematoma (SDH), subarachnoid hemorrhage (SAH), intraventricular hemorrhage (IVH), brain contusions, intracerebral hematoma (ICH), fissure fractures, depressed fractures, and pellets. Fourteen patients (14.7\%) had multiple pathologies on CT and 6 patients (6.3\%) had normal CT brain. Figure 1 shows the initial CT findings of our patients.

Based on the clinical assessment and the CT findings, surgical intervention was performed for 22 patients (23.2\%). Nine patients were operated upon for evacuation of an intracranial hematoma being extradural in 8 patients and subdural in one patient. Another 11 patients were operated upon for elevation of depressed fractures; 9 patients with compound fractures and 2 patients with simple fractures. Patients with simple depressed fractures were operated upon due to cosmetic deformity in one patient and due to the presence of neurological deficit in the other patient. Decompressive craniotomy was performed for one patient with subdural hematoma and hemispherical edema with midline shift and another patient was operated upon for growing skull fracture. Patients operated upon for non-neurosurgical injuries and patients operated upon for only closure of scalp wounds were not included in the surgical group.

The length of hospital stay ranged between one day and 31 days, with mean length of hospital stay being 4 days. One patient was transferred to another hospital few hours after admission, and was excluded from length of hospital stay and clinical outcome assessment. Figure 2 shows the length of hospital stay in our patients. Based on GOS-E Peds, there were 83 patients (88.3\%) with good recovery (GOS-E Peds 1, 2), 7 patients (7.4\%) with moderate disability (GOS-E Peds3, 4), and 4 patients (4.3\%) died (GOS-E Peds 8). One mortality occurred in a patient with severe head injury and associated lung contusion who was operated upon by urgent evacuation of a large parietal extradural hematoma and died 4 days later from the associated lung contusion. Another patient with liver injury and intraperitoneal hemorrhage died few hours following laparotomy. The other 2 patients had severe head injury with no surgical lesion on the CT and were admitted to the intensive care where they both died 3 and 4 days after admission. 


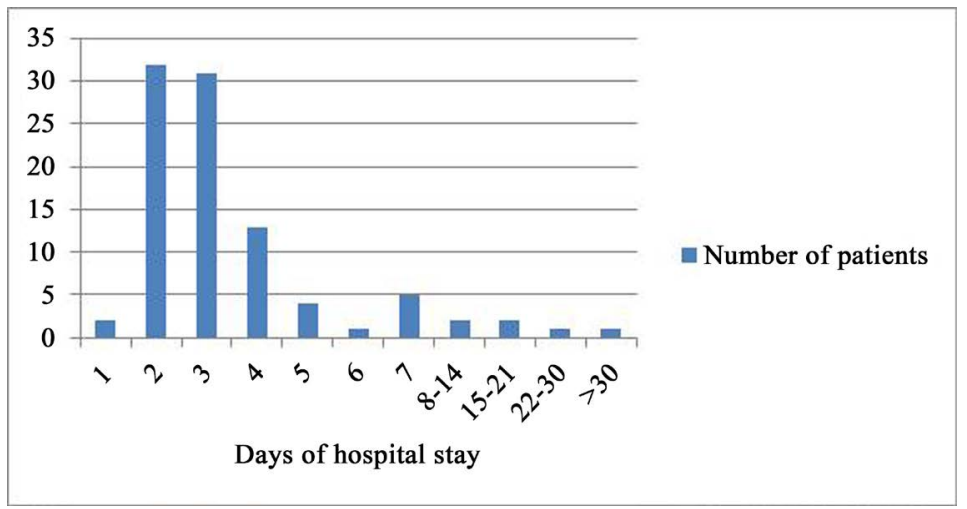

Figure 2. This figure shows the length of hospital stay.

\section{Discussion}

Head injuries are very common in infants and children, but are mostly minor and require no medical care. However, a mild head injury that may appear insignificant can sometimes lead to intracranial complication and deterioration. Although long-term motor and cognitive dysfunction occur more commonly in the severely injured children, deficits can occur even to those children with mild and moderate head injury. In general, degree of recovery from head injuries is better in infants and children than in adults [5] [13].

Our series showed male predominance with male to female ratio of $1.5: 1$ (60\% males and $40 \%$ females). Adamo et al. also had male predominance where $56 \%$ of his accidental cases were boys [14]. On their report of 29 infants with head trauma, Vaghani et al. had 17 boys (59\%) and 12 girls (41\%) [7].

Falls represented the most common mode of trauma in our patients (82.1\%), followed by trauma to the head with blunt or sharp objects (13.7\%). Both road traffic accidents and pellets injury accounted for less than $5 \%$ of cases. According to the literature, falls are the most common causes of head injury in toddlers and children below 4 years of age, accounting for about 75\% of accidental injuries [15] [16]. Vaghani et al. reported the mode of trauma being fall from height in 93\% and road traffic accidents in 7\% [7].

Another important cause of head injury in infants is nonaccidental head trauma due to child abuse which is known as "shaken baby syndrome". Many articles addressed nonaccidental head trauma reporting its incidence to range between $1.3 \%$ and $25 \%$ of all cases of infantile head trauma admitted to the hospital [8] [14] [17]. This wide range may be explained by the frequent under-diagnosis of this mode of trauma which should be suspected when there are findings suggestive of traumatic brain injury with no history of trauma or inappropriate history to the degree of injury observed. Findings include the presence of subdural hematoma on CT or magnetic resonance imaging (MRI), particularly in the interhemispheric fissure or over the tentorium in addition to retinal hemorrhage on ophthalmological examination [17] [18]. Although nonaccidental head trauma was not considered as an exclusion criterion, our study did not include any patient with proved nonaccidental head trauma.

The majority of our patients (84.2\%) had mild head injury, with only 5 patients (5.3\%) with severe head injury. In their series including infants and toddlers below 3 years of age with accidental and non-accidental head trauma, Adamo et al. reported that $86.5 \%$ of their patients with accidental head trauma had mild head injury, with 5.4\% of their accidental patients with severe head injury [14]. In their series of 233 infants with isolated head trauma, Brown et al. mentioned that $97 \%$ of these patients had minor head injury [8]. Vaghani et al. had mild head injury in $62 \%$ of their patients, moderate injury in $14 \%$, and severe injury in $24 \%$ [7].

Fifty six patients (59\%) had skull fractures on their CT brain, being fissure fracture in 39 patients and depressed fractures in 19 patients, including 2 patients having both fissure fracture and depressed fracture. One patient with fissure fracture on the initial CT developed growing skull fracture later, and was operated upon. Skull fractures are common in infants as the bone is relatively thin and can break easily after an impact. These fractures can be associated with growing skull fracture which is a late complication of injury, usually occurring in children below 2 years of age [13]. Adamo et al. reported skull fractures in 57\% of their patients with accidental head trauma [14].

Subgaleal hematomas are common in children and they may be huge with significant blood loss in the subgaleal space that may require blood transfusion in infants below 1 year of age [19]. We had 31 patients with sub- 
galeal hematomas which were huge in 7 patients resulting in anemia requiring blood transfusion. Thirty two patients (33.7\%) had intracranial hematomas being extradural in $13.7 \%$, subdural in $11.6 \%$, subarachnoid in $5.3 \%$, intracerebral in $2.1 \%$ and intraventricular in $1 \%$. Intracranial hematomas are a relatively uncommon sequel of head injury in children, with extradural hematomas being the most common in some series [20] [21]. Adamo et al. reported intracranial hematomas in $35.1 \%$ of their patients with accidental trauma, being extradural in $6.2 \%$, subdural in $14.5 \%$, subarachnoid in $7.7 \%$, and intraparenchymal in $6.7 \%$. They had $7.8 \%$ of their patients with CT showing no abnormalities compared to $6.3 \%$ in our series [14]. Vaghani et al. had subdural hematoma in $27 \%$ of their patients being the most common injury [7].

Although several authors highlighted the use of cranial ultrasound (US) in diagnosis of skull fractures and traumatic intracranial hemorrhage in infants and children to avoid the hazards of CT including radiation and possible need for sedation [22] [23], our policy was to perform CT brain for all admitted patients with head trauma even if the trauma was mild and the patient was conscious. We did not use cranial US due to its unavailability on urgent basis at all times. Fundaro and colleagues concluded that performing CT even in children with mild injuries especially if there was loss of consciousness or scalp swelling is justified by the abnormal findings detected in a proportion of these patients [24].

Based on the pediatric extended GOS (GOS-E Peds), 88.3\% of patients had good recovery (GOS-E Peds 1, 2), 7.4\% had moderate disability (GOS-E Peds 3, 4), and 4 patients died (4.3\%). Vaghani et al. had 69\% of their patients with good recovery (GOS 5), 10.3\% with moderate disability (GOS 4), 10.3\% with severe disability (GOS 3), and 10.3\% died (GOS1) [7]. Adamo et al. assessed clinical outcome based on KOSCHI scale (King's Outcome Scale for Childhood Head Injury) for outcome assessment [25]. They had good recovery in $80.4 \%$ of patients with accidental trauma, moderate disability in $15.8 \%$, and mortality in $0.6 \%$ of patients [14].

The limitations of the study include the relatively small number of patients, the possible underestimation of non-accidental head trauma, and the lack of long term follow up of these patients. Further studies are recommended with larger sample size and longer follow up period, with special emphasis on nonaccidental head trauma.

\section{Conclusion}

Most of head injuries in infants are mild. The commonest etiology is falls, which is a preventable cause in many circumstances. The majority of these patients do not require surgical intervention. Good recovery is the rule in most of these infants with low mortality rates.

\section{References}

[1] Gerber, P. and Coffman, K. (2007) Nonaccidental Head Trauma in Infants. Child's Nervous System, 23, 499-507. http://dx.doi.org/10.1007/s00381-006-0267-4

[2] Manwaring, J. and Adelson, P.D. (2012) Management of Pediatric Severe Traumatic Brain Injury. In: Quinones-Hinojosa, A., Ed., Schmidek \& Sweet Operative Neurosurgical Techniques, Elsevier Saunders, Philadelphia, 741-752. http://dx.doi.org/10.1016/B978-1-4160-6839-6.10062-0

[3] Adelson, P.D. and Kochanek, P.M. (2000) Head Injury in Children. In: Kaye, A.H. and Black, P.Mcl., Eds., Operative Neurosurgery, Churchill Livingstone, London, 279-290.

[4] Keenan, H.T., Runyan, D.K., Marshall, S.W., Nocera, M.A., Merten, D.F. and Sinal, S.H. (2003) A Population-Based Study of Inflicted Traumatic Brain Injury in Young Children. JAMA, 290, 621-626. http://dx.doi.org/10.1001/jama.290.5.621

[5] Giza, C.C. (2006) Lasting Effects of Pediatric Traumatic Brain Injury. Indian Journal of Neurotrauma (IJNT), 3, 19-26. http://dx.doi.org/10.1016/S0973-0508(06)80005-5

[6] Adamo, M.A., Drazin, D. and Waldman, J.B. (2009) Decompressive Craniectomy and Postoperative Complication Management in Infants and Toddlers with Severe Traumatic Brain Injuries. Journal of Neurosurgery: Pediatrics, 3, 334-339. http://dx.doi.org/10.3171/2008.12.PEDS08310

[7] Vaghani, G., Singh, P.K., Gupta, D.K., Agrawal, D., Sinha, S., Satyarthee, G., et al. (2013) Outcome of Patients with Traumatic Head Injury in Infants: An Institutional Experience at Level 1 Trauma Center. Journal of Pediatric Neurosciences, 8, 104-107. http://dx.doi.org/10.4103/1817-1745.117836

[8] Brown, C.W., Akbar, S.P. and Cooper, J.G. (2014) Things That Go Bump in the Day or Night: The Aetiology of Infant Head Injuries Presenting to a Scottish Paediatric Emergency Department. European Journal of Emergency Medicine, 


\section{1, 447-450. http://dx.doi.org/10.1097/MEJ.0000000000000125}

[9] Teasdale, G. and Jennett, B. (1974) Assessment of Coma and Impaired Consciousness: A Practical Scale. The Lancet, 2, 81-84. http://dx.doi.org/10.1016/S0140-6736(74)91639-0

[10] James. H., Anas, N. and Perkin, R.M. (1985) Brain Insults in Infants and Children. Grune \& Stratton, New York.

[11] Bullock, M.R., Chesnut, R., Ghajar, J., Gordon, D., Hartl, R., Newell, D.W., et al. (2006) Guidelines for the Surgical Management of TBI. Neurosurgery, 58, S2-1-62.

[12] Beers, S.R., Wisniewski, S.R., Garcia-Filion, P., Tian, Y., Hahner, T., Berger, R.P., et al. (2012) Validity of a Pediatric Version of the Glasgow Outcome Scale-Extended. Journal of Neurotrauma, 29, 1126-1139. http://dx.doi.org/10.1089/neu.2011.2272

[13] Jernigan, S. and Proctor, M.R. (2011) Management of Severe Head Injury in Children. In: Winn, H.R., Ed., Youmans Neurological Surgery, Elsevier Saunders, Philadelphia, 2166-2175. http://dx.doi.org/10.1016/B978-1-4160-5316-3.00212-4

[14] Adamo, M.A., Drazin, D., Smith, C. and Waldman, J.B. (2009) Comparison of Accidental and Nonaccidental Traumatic Brain Injuries in Infants and Toddlers: Demographics, Neurosurgical Interventions, and Outcomes. Journal of Neurosurgery: Pediatrics, 4, 414-419. http://dx.doi.org/10.3171/2009.6.PEDS0939

[15] Aldrich, E.F., Levin, H.S. and Eisenberg HM. (1996) Mild Head Injury in Children. In: Youmans, J.R., Ed., Neurological Surgery, WB Saunders, Philadelphia, 1719-1729.

[16] Agran, P.F., Anderson, C., Winn, D., Trent, R., Walton-Haynes, L. and Thayer, S. (2003) Rates of Pediatric Injuries by 3-Month Intervals for Children 0 to 3 Years of Age. Pediatrics, 111, 683-692. http://dx.doi.org/10.1542/peds.111.6.e683

[17] Duhaime, A.C. and Christian, C.W. (2011) Child Abuse. In: Winn, H.R., Ed., Youmans Neurological Surgery, Elsevier Saunders, Philadelphia, 2176-2183. http://dx.doi.org/10.1016/B978-1-4160-5316-3.00213-6

[18] Duhaime, A.C., Christian, C.W., Rorke, L.B. and Zimmerman, R.A. (1998) Nonaccidental Head Injury in Infants-The “Shaken-Baby Syndrome”. The New England Journal of Medicine, 338, 1822-1829. http://dx.doi.org/10.1056/NEJM199806183382507

[19] Kilani, R.A. and Wetmore, J. (2006) Neonatal Subgaleal Hematoma: Presentation and Outcome—Radiological Findings and Factors Associated with Mortality. American Journal of Perinatology, 23, 41-48. http://dx.doi.org/10.1055/s-2005-923438

[20] Luerssen, T.G., Klauber, M.R. and Marshall, L.F. (1988) Outcome from Head Injury Related to Patient’s Age. A Longitudinal Prospective Study of Adult and Pediatric Head Injury. Journal of Neurosurgery, 68, 409-416. http://dx.doi.org/10.3171/jns.1988.68.3.0409

[21] Mohanty, A., Kolluri, V.R.S., Subbakrishna, D.K., Satish, S., Mouli, B.A. and Das, B.S. (1995) Prognosis of Extradural Hematomas in Children. Pediatric Neurosurgery, 23, 57-63. http://dx.doi.org/10.1159/000120936

[22] Rabiner, J.E., Friedman, L.M., Khine, H., Avner, J.R. and Tsung, J.W. (2013) Accuracy of Point-of-Care Ultrasound for Diagnosis of Skull Fractures in Children. Pediatrics, 131, 1757-1764. http://dx.doi.org/10.1542/peds.2012-3921

[23] McCormick, T., Chilstrom, M., Childs, J., McGarry, R., Seif, D., Mailhot, T., et al. (2015) Point-of-Care Ultrasound for the Detection of Traumatic Intracranial Hemorrhage in Infants: A Pilot Study. Pediatric Emergency Care. http://dx.doi.org/10.1097/PEC.0000000000000518

[24] Fundarò, C., Caldarelli, M., Monaco, S., Cota, F., Giorgio, V., Filoni, S., et al. (2012) Brain CT Scan for Pediatric Minor Accidental Head Injury. An Italian Experience and Review of Literature. Child's Nervous System, 28, 10631068. http://dx.doi.org/10.1007/s00381-012-1717-9

[25] Crouchman, M., Rossiter, L., Colaco, T. and Forsyth, R. (2001) A Practical Outcome Scale for Paediatric Head Injury. Archives of Disease in Childhood, 84, 120-124. http://dx.doi.org/10.1136/adc.84.2.120 